\title{
Activity of Upper Body Muscles during Bowing and Prostration Tasks in Healthy Subjects
}

M.K.M. Safee, W.A.B. Wan Abas, N.A. Abu Osman, and F. Ibrahim

INTRODUCTION

The electrical activity in the human muscles can be measured using electromyography (EMG). This allows for the measurement of the change in the membrane potential as the action potentials are transmitted along the fiber. The study of the muscles from this perspective can be valuable in providing information concerning the control of voluntary and reflexive movement. The study of muscle activity during a particular task can yield insight into which muscles are active and when the muscles initiate and cease their activities.

Surface electrodes are placed on the skin over a muscle and thus are mainly used for superficial muscle [1]. With the technique of electromyography, or recording the electrical impulses generated by muscular contractions, it is possible to determine very precisely which muscle, superficial and deep, contract during a given movement. Electromyography can also provide information on the sequence in which each of several participating muscles contracts and can help in estimating the strength of contraction of each muscle [2].

There are many research findings that show the benefits of muscle contraction and muscle strength. For example, to protect and stabilize the head and neck in high Gz environments, higher neck muscle strength is needed; less muscle strength in fighter pilot may cause pain and perhaps reduced mission effectiveness [3]. Besides, more research that relate 
the NE to the FRP and low back pain (LBP) [4-6]. The FRP

is defined by a reduction in or silence of myoelectric activity

of the lumbar erector spine muscle observed during full

trunk flexion [5]. The FRP was found to occur at 40。 to 70o

of body flexion [7, 8]. Based on the biomechanical models

of the spine, it was proposed that spinal stabilization should

be considered the result of highly-coordinated muscular

activation interacting with passive elements [9]. Spinal

stability is also highly dependent on spinal load and posture

[10] as well as task requirements [12]. Instability of the

lumbar spine has been suggested to be both a cause and a

consequence of LBP [9].

Efficiency of motion and stresses imposed on the spine

are very much dependent upon the posture maintained in the

trunk as well as on the trunk stability. Positioning of the

vertebral segments is so important that a special focus on

posture and spinal stabilization is warranted [1]. Muscles

that play an important role in spinal stabilization include the

transverse abdominal, multifidus, erector spine, and internal

oblique [1]. The erector spine is better suited for control of

spinal orientation by nature of its ability to produce extension

[12]. The RA is one of the abdominal wall's muscles.

The wall is very important because it not only contract to

increase intra-abdominal pressure but also distend considerably,

accommodating expansions caused by ingestion,

pregnancy, fat deposition, or pathology [13]. Beside that, there are many experiments that show muscle contraction

for the biceps and triceps during exercise $[14,15]$. Other

experiments also show that the TRP and DT are also involved

in shoulder exercise $[16,17]$.

Common exercise recommendations from health professionals

include trunk exercises to prevent and treat low back

injuries. Knowing the trunk muscle activation levels during

exercises is important in the prescription and design of

exercise programs that aim to increase the training intensity

over time (progressive resistance model). Previous researches

have documented trunk muscle electromyograms 
(EMGs) during various exercises designed to train the trunk musculature and during functional activities [18-21]. Ng et al [20] found that abdominal and trunk muscles not only produce torque but also maintain spinal posture and stability during axial rotation exertions.

Exercise programming for a young, healthy population incorporates exercises that push the muscular system to high levels of performance. Muscles can exert force and develop power to produce the desired movement outcomes. The lost of strength in the muscles can create a variety of problems, ranging from inability to reach overhead or open a jar lid to difficulty using stairs and getting up out of a chair [1]. Skeletal muscle performs a variety of different function, all of which are important to efficient performance of the human body. The three functions relating specifically to human movement are contributing to the production of skeletal movement, assisting in joint stability, and maintaining posture and body position [1].

From the current research, it is possible to identify the muscles that are involved and contract during bowing and prostration. The experiment can also serve as a pilot study on the biomechanical response of human muscle during the Muslims' prayer, or the salat.

\section{SUBJECTS AND METHODS}

Subjects

A total of 11 male undergraduates (age: $23 \pm 5.1$ years) with no medical history and no back pain were recruited as subjects of the study. Subjects were verbally informed about the experimental protocols, and they read and signed a consent form prior to participating in the experiments. Three repetitions were recorded for every salat and exercise protocol. 
Apparatus

Disposable bipolar $\mathrm{Ag}-\mathrm{AgCl}$ disc surface electrodes with a diameter of one $\mathrm{cm}$ were affixed over the chosen muscle groups, parallel to their fiber orientation in the muscle belly. The electrodes were connected to an EMG data collection system (Myomonitor IV Wireless Transmission, Delys) and the signals were collected using customized software (Delys EMGWorks, Boston, MA, USA). These records were then downloaded into a personal computer (Toshiba, Japan). The EMG bandwidth was $20-450 \mathrm{~Hz}$ at a sampling rate $1500 \mathrm{~Hz}$.

The electrodes were placed according to the SENIAM recommendation [22]. The myomonitor was capable of recording 16 muscles simultaneously.

Experimental Procedure

The current study involved EMG recording of eight muscles of each subject while the subjects were performing the prostration and bowing actions. The eights muscles are the neck extensors (NE), sternocleidomastoideus (SCM), trapezius (TRP), deltoid (DT), biseps brachii (BB), triceps brachii (TB), rectus abdominus (RA), and erector spine (ES), Figure 1. At each site, the readings were taken twice and the average of the two values was recorded for analysis. 11 subjects were involved in the study.

Full text is available at :

http://link.springer.com/chapter/10.1007/978-3-642-21729-6_34 